\title{
Biodistribution and Pharmacokinetics of $O$-Palmitoyl Tilisolol, a Lipophilic Prodrug of Tilisolol, after Intravenous Administration in Rats
}

\author{
Shigeru Kawakami, ${ }^{*}, a$ Nao Ohshima, ${ }^{b}$ Ryu Hirayama, ${ }^{a}$ Masami Hirai, ${ }^{b}$ Takashi Kitahara, ${ }^{b}$ \\ Toshiyuki Sakaeda, ${ }^{c}$ Takahiro MukaI,${ }^{a}$ Koyo Nishida, ${ }^{a}$ Junzo NaKamura, ${ }^{a}$ Mikiro NaKashima, ${ }^{b}$ and \\ Hitoshi SASAKI ${ }^{b}$ \\ ${ }^{a}$ School of Pharmaceutical Sciences, Nagasaki University; 1-14 Bunkyo-machi, Nagasaki 852-8521, Japan: ${ }^{b}$ Department \\ of Hospital Pharmacy, Nagasaki University School of Medicine; 1-7-1 Sakamoto, Nagasaki 852-8501, Japan: and \\ ${ }^{c}$ Department of Hospital Pharmacy, School of Medicine, Kobe University; 7-5-2 Kusunoki-cho, Chuo-ku, Kobe 650-0017, \\ Japan. Received January 28, 2002; accepted April 22, 2002
}

\begin{abstract}
The purpose of this study was to modify the biodistribution and pharmacokinetics of tilisolol, a $\beta$-blocker, using the palmitoyl prodrug approach. After intravenous administration of tilisolol and $O$-palmitoyl tilisolol in rats, drug concentrations were determined in blood, bile, urine, and several tissues. The concentration-time profiles of tilisolol and $O$-palmitoyl tilisolol were analyzed pharmacokinetically. The blood concentrations of $O$ palmitoyl tilisolol after intravenous administration of $O$-palmitoyl tilisolol were about 10 -fold higher than those of tilisolol after intravenous administration of tilisolol. The biliary excretion rates of $O$-palmitoyl tilisolol and tilisolol after intravenous administration of $O$-palmitoyl tilisolol were about 10- to 100 -fold larger than those of tilisolol after intravenous administration of tilisolol. In addition, the hepatic uptake clearance of $O$-palmitoyl tilisolol after intravenous administration of $O$-palmitoyl tilisolol was 3.6-fold higher than that of tilisolol after the intravenous administration of tilisolol. In the in vitro experiments, it was demonstrated that the distribution ratios between blood cells and plasma (blood/plasma) of $O$-palmitoyl tilisolol and tilisolol was 95.7 and $55.5 \%$, respectively. These findings suggest that $O$-palmitoyl tilisolol exists as a binding form with biological components, especially blood cells, in systemic circulation. In conclusion, the palmitoyl prodrug approach is useful as a drug delivery system to deliver the parent drug to the liver.
\end{abstract}

Key words prodrug; tilisolol; pharmacokinetic; liver; $\beta$-blockers; stability

In medical treatment, it is necessary to control the behavior of drugs for effective treatment, and many attempts have been made to deliver drugs to target sites by means of drug delivery systems. Recently, the use of high-density lipoprotein, ${ }^{1)}$ low-density lipoprotein (LDL), ${ }^{2,3)}$ liposomes, ${ }^{4,5)}$ and lipid emulsions ${ }^{5}$ ) as drug carriers have been focused on to control drug delivery. However, it is difficult to load hydrophilic drugs to these hydrophobic carriers because of their poor affinity. Even if the drug carrier exhibited a favorable disposition profile, a rapid release of the incorporated drug often leads to failure to achieve therapeutic potency. ${ }^{4,6)}$

Sasaki et al. ${ }^{5)}$ and Tokunaga et al. ${ }^{7)}$ demonstrated the drug delivery of mitomycin $\mathrm{C}$ lipophilic prodrugs using liposomes and/or lipid emulsions. Sqalli-Houssaini et al. ${ }^{8)}$ reported the drug delivery of lipophilic prodrugs for anti-HIV drugs to macrophages via LDL pathways. An effective approach to enhance the affinity of drugs to these hydrophobic carriers might be using highly lipophilic prodrugs. Therefore, it is important to evaluate the biodistribution and pharmacokinetics of highly lipophilic prodrugs after intravenous administration to develop these lipid carrier systems; however, little such information has been obtained.

We previously synthesized the $O$-palmitoyl ester prodrug of tilisolol ( $O$-palmitoyl tilisolol) and it showed high lipophilicity compared with tilisolol. ${ }^{9,10)}$ In the present study, we evaluated the biodistribution and pharmacokinetics after intravenous administration of $O$-palmitoyl tilisolol in rats. Tilisolol, which is a $\beta$-blocker, was used as a model drug because of its easy detection with fluorescence. ${ }^{11)}$

\section{MATERIALS AND METHODS}

Materials Tilisolol hydrochloride was supplied from Nisshin Flour Milling Co., Ltd. (Tokyo, Japan). All other chemicals of reagent grade were obtained from Nacalai Tesque, Inc. (Kyoto, Japan). Phosphate-buffered saline (PBS, $\mathrm{pH}$ 7.4) was prepared by mixing isotonic phosphate buffer with an equal volume of $0.9 \% \mathrm{NaCl}$.

Synthesis of $\boldsymbol{O}$-Palmitoyl Tilisolol $O$-Palmitoyl tilisolol was synthesized according to a method reported previously. $^{10)}$

Drug Distribution Ratio between Blood Cells to Plasma The drug distribution to blood cells was calculated from blood concentration and plasma concentration. Drugs ( $0.005 \mu \mathrm{mol} ; 50 \mu \mathrm{l})$ dissolved by ethanol were added to rat whole blood $(950 \mu \mathrm{l})$ and were incubated at $37^{\circ} \mathrm{C}$ for $30 \mathrm{~min}$. At first, the blood was centrifuged at $12000 \boldsymbol{g}$ for $5 \mathrm{~min}$. The drug concentration in blood cells was determined after removal of the plasma. The plasma was ultrafiltrated using a micropartition system (Ultrafree ${ }^{\circledR}-\mathrm{C} 3$, Millipore, Co., Bedford, MA, U.S.A.) at $5000 \mathrm{~g}$ for $10 \mathrm{~min}$. The drug concentration in the preparation $\left(C_{\mathrm{T}}\right)$ and that in the ultrafiltrate $\left(C_{\mathrm{W}}\right)$ were assayed by HPLC. Thus, the equation for the drug binding ratio to protein was as follows:

$$
\text { binding ratio (\%) }=\left(\frac{C_{\mathrm{T}}-C_{\mathrm{W}}}{C_{\mathrm{T}}}\right) \cdot 100
$$

Stability Experiment The blood, plasma and tissues (liver, kidney, lung, spleen, and heart) for the stability experiments were obtained from rats. The tissues were homogenized on ice in $1.17 \% \mathrm{KCl}$ with a glass-teflon homogenizer, and centrifuged at $800 \boldsymbol{g}$ for $10 \mathrm{~min}$. The supernatants were 
diluted to $10 \mathrm{mg}$ protein $/ \mathrm{ml}$ with $1.17 \% \mathrm{KCl}$. The protein concentrations were determined by Bio-Rad protein assay (Bio-Rad Laboratories, Hercules, U.S.A.). The stability experiments of $O$-palmitoyl tilisolol were performed at $37^{\circ} \mathrm{C}$. At appropriate time intervals, aliquots of the solution were withdrawn and subjected to HPLC assay.

In Vivo Experiment Animal experiments conformed to the Guidelines for Animal Experimentation at Nagasaki University.

Male Wistar rats $(220-260 \mathrm{~g})$ were anesthetized with sodium pentobarbital $(50 \mathrm{mg} / \mathrm{kg}$, i.p. $)$, and the left femoral artery was cannulated with a polyethylene tube $(0.5 \mathrm{~mm}$ i.d., $0.8 \mathrm{~mm}$ o.d.; Dural Plastics, Dural, Australia). A $3 \mathrm{~cm}$ incision was made in the middle abdomen, and the common bile duct was cannulated with a polyethylene tube $(0.28 \mathrm{~mm}$ i.d., $0.61 \mathrm{~mm}$ o.d.; Becton Dickinson, Parsippany, NJ, U.S.A.). The body temperature of the rats was maintained at $37^{\circ} \mathrm{C}$ with a heat lamp during the experiment. $O$-Palmitoyl tilisolol and tilisolol $(6 \mu \mathrm{mol} / \mathrm{kg})$ were injected into the jugular vein. Then, blood and bile were collected at predetermined time points. Urine was completely collected at $180 \mathrm{~min}$ after intravenous administration and diluted 100-folds with distilled water. The rats were sacrificed by an overdose of sodium pentobarbital at 30 and $180 \mathrm{~min}$ after intravenous administration, and several organs (liver, kidney, lung, spleen, and heart) were then excised. The tissues were weighed and homogenized in an appropriate volume of $1.17 \% \mathrm{KCl}$. Aliquots of blood, bile, urine, and tissue homogenates were used for the HPLC assay.

Drug Assay for Biological Samples Biological samples (200 $\mu \mathrm{l}$ for plasma, bile, and urine; $1.5 \mathrm{ml}$ for tissue homogenates) were shaken with $7 \mathrm{ml}$ of diethyl ether for $15 \mathrm{~min}$, and thereafter centrifuged at $800 \mathrm{~g}$ for $10 \mathrm{~min}$. At first, organic layers containing $O$-palmitoyl tilisolol were evaporated. The residues were dissolved in a mixture of $0.1 \mathrm{M}$ $\mathrm{HCl}$ of ethanol $(150 \mu \mathrm{l})$ and methanol $(150 \mu \mathrm{l})$ containing an internal standard $(0.03 \mu \mathrm{l} / \mathrm{ml}$ benzyl salicylate). The mixtures were centrifuged at $12000 \mathrm{~g}$ and $80 \mu \mathrm{l}$ of the supernatant was injected into the HPLC. For the assay of tilisolol, the aqueous layers were extracted again with diethyl ether $(6 \mathrm{ml})$ after the addition of $0.5 \mathrm{M} \mathrm{NaOH}$. The organic layers containing tilisolol were evaporated, then the residues were dissolved in $0.1 \mathrm{M} \mathrm{HCl}(100 \mu \mathrm{l})$ and methanol $(150 \mu \mathrm{l})$ containing an internal standard $(300 \mu \mathrm{g} / \mathrm{ml} o$-ethoxybenzamide). The mixtures were centrifuged at $12000 \boldsymbol{g}$ for $10 \mathrm{~min}$ and $80 \mu \mathrm{l}$ of the supernatant was injected into the HPLC. The recovery percentage for the extraction of tilisolol and $O$-palmitoyl tilisolol from blood and each tissue homogenate was approximately $75 \%$. The detection limit of tilisolol in the blood and in each tissue homogenate was $0.06 \mu \mathrm{M}$. Similarly, the detection limit of $O$-palmitoyl tilisolol in the blood and each tissue homogenate was $0.6 \mu \mathrm{M}$.

HPLC An HPLC system (LC-6A, Shimadzu Co., Ltd., Kyoto, Japan) with a fluorescence spectromonitor (RF-530, Shimadzu Co., Ltd., Kyoto, Japan) was used in reverse phase mode. The excitation wavelength and the emission wavelength were monitored at 315 and $420 \mathrm{~nm}$, respectively. The stationary phase used was a Cosmosil $5 \mathrm{C}_{18}-\mathrm{P}$ packed column (150 mm length $\times 4.6 \mathrm{~mm}$ i.d., Nacalai Tesque, Inc., Kyoto, Japan). For analysis of tilisolol, the mobile phase was methanol: $50 \mathrm{~mm} \mathrm{NaH} \mathrm{PO}_{4}(39: 61, \mathrm{v} / \mathrm{v})$ at a flow rate of
$1.0 \mathrm{ml} / \mathrm{min}$. The retention times of tilisolol and $o$-ethoxybenzamide were 4.5 and $11.5 \mathrm{~min}$, respectively. For analysis of $O$-palmitoyl tilisolol, the mobile phase was 2-propanol : acetonitrile : $50 \mathrm{~mm} \mathrm{NaH} \mathrm{PO}_{4}(37: 33: 30, \mathrm{v} / \mathrm{v})$ at a flow rate of $1.0 \mathrm{ml} / \mathrm{min}$. The retention times of $O$-palmitoyl tilisolol and benzyl salicylate were 10.8 and $4.4 \mathrm{~min}$, respectively.

Pharmacokinetic Analysis Blood profiles of drugs after intravenous administration were analyzed using a two-compartment model. $V_{\mathrm{c}}$ and $V_{\mathrm{p}}$ are the distribution volumes of the central compartment and the peripheral compartment. $K_{\mathrm{el}}$ is the first order elimination rate constant from the central compartment. $K_{12}$ and $K_{21}$ are the first order transfer rate constants between the central compartment and the peripheral compartment. Pharmacokinetic parameters were calculated by a nonlinear least squares computer program, MULTI. ${ }^{12)}$ This program was written by BASIC and run on a personal computer (PC-9821 V13, NEC, Tokyo, Japan).

Tissue distribution data were evaluated using tissue uptake clearances as reported previously. ${ }^{13)}$ The change of the drug amount in tissue with time can be described as follows:

$$
\frac{d T(t)}{d t}=C l_{\text {in }} \cdot C(t)-K_{\text {out }} \cdot T(t)
$$

where $T(t)$ is the amount of drugs in $1 \mathrm{~g}$ tissue, $C(t)$ is the blood concentration of drugs, $C l_{\text {in }}$ is the tissue uptake rate index (clearance) from blood to tissue, and $K_{\text {out }}$ is the efflux rate constant from the tissues. To estimate the uptake rate index, distribution in the first $30 \mathrm{~min}$ after intravenous administration was measured, allowing the contribution of efflux processes and metabolism to be ignored. If efflux is ignored, Eq. 1 is integrated as follows:

$$
C l_{\text {in }}=\frac{T(t)}{\int_{0}^{t} C(t) d t}=\frac{T(t)}{A U C_{0-\mathrm{t}}}
$$

According to Eq. 2, the tissue uptake rate index is calculated using the amount of drugs in tissue at $30 \mathrm{~min}$ and the area under the blood concentration-time curve (AUC) up to the time obtained through curve fitting to the blood concentration-time course based on a two-compartment model. Tissue uptake clearance $\left(C L_{\text {tissue }}\right)$ is expressed as follows:

$$
C L_{\text {tissue }}=C l_{\text {in }} \cdot W
$$

where $W(\mathrm{~g})$ is the total weight of the tissues.

Statistical Analysis Statistical comparisons were performed by analysis of variance. $p<0.05$ was considered to be indicative of statistical significance.

\section{RESULTS AND DISCUSSION}

Figures $1 \mathrm{~A}$ and $\mathrm{B}$ shows the stability of $O$-palmitoyl tilisolol in blood, plasma, and bile at $37^{\circ} \mathrm{C}$. $O$-Palmitoyl tilisolol was rapidly degraded in plasma and bile, and tilisolol was appeared. The conversion of $O$-palmitoyl tilisolol to tilisolol was also observed in blood. In our previous study, $O$-palmitoyl tilisolol was converted to tilisolol even in $40 \%$ ethanol of PBS, and its half life was $66.6 \pm$ 3.4 min. ${ }^{10}$ ) This result suggested that chemical catalysis contributes to the conversion of $O$-palmitoyl tilisolol to tilisolol. In contrast, $O$-palmitoyl tilisolol was gradually degraded in various tissue homogenates (Figs. 1C, D). Thus, the drug- 

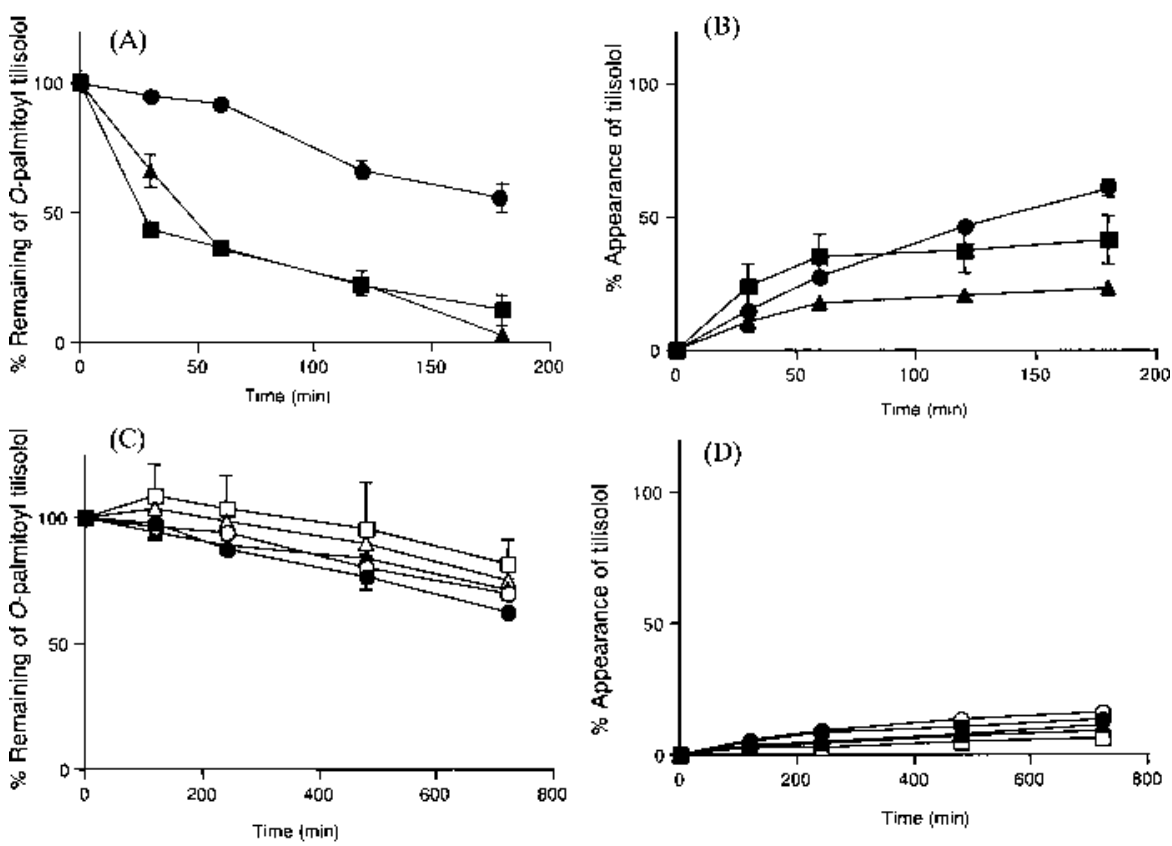

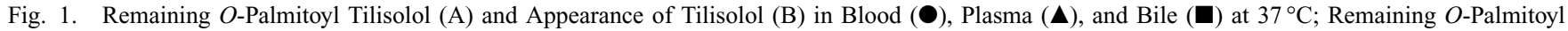

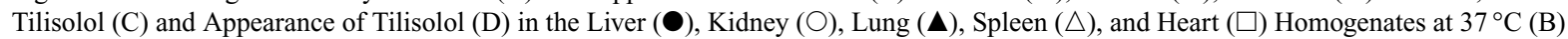

Each point represents the mean \pm S.E. of at least three experiments.

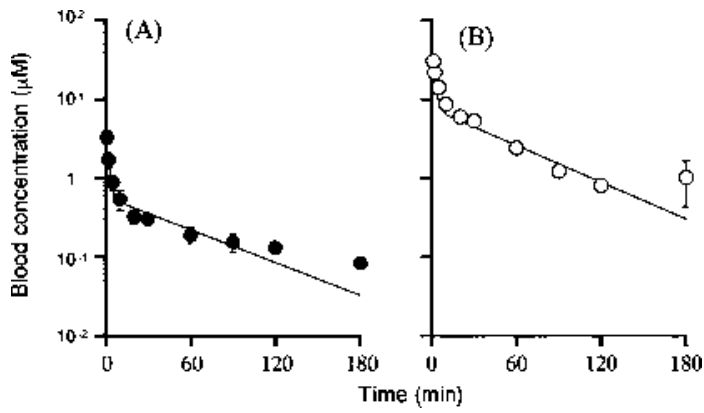

Fig. 2. Blood Concentration of Tilisolol (A) and $O$-Palmitoyl Tilisolol (B) after Intravenous Administration at a Dose of $6 \mu \mathrm{mol} / \mathrm{kg}$ in Rats

-) and $(O)$ indicate tilisolol and $O$-palmitoyl tilisolol, respectively. Fitting line calculated according to a two compartment model. Each point represents the mean \pm S.E. of at least three experiments.

protein binding in the tissue homogenates may cause a suppression of the degradation of lipophilic prodrugs. ${ }^{14)}$ In the $40 \%$ ethanol of PBS, blood and each tissue homogenate, tilisolol appeared to correspond to the degradation extent of $O$-palmitoyl tilisolol. However, the extent of tilisolol appearing in bile and plasma was lower than the degradation extent of $O$-palmitoyl tilisolol. These results suggest that $O$-palmitoyl tilisolol might be degraded not only to tilisolol but also to other metabolites in bile and plasma.

The drug concentration profiles in blood after the intravenous administration of tilisolol and $O$-palmitoyl tilisolol are shown in Fig. 2. Blood concentrations of $O$-palmitoyl tilisolol after the intravenous administration of $O$-palmitoyl tilisolol were approximately 10-fold higher than that of tilisolol after the intravenous administration of tilisolol. Tilisolol was not detected in blood after the intravenous administration of $O$-palmitoyl tilisolol. The blood concentration profiles of tilisolol and $O$-palmitoyl tilisolol after the intra-
Table 1. Pharmacokinetic Parameters of Tilisolol and $O$-Palmitoyl Tilisolol after Intravenous Administration in Rats

\begin{tabular}{|c|c|c|}
\hline \multirow[b]{2}{*}{ Parameters } & $\stackrel{K_{12}}{\longrightarrow}$ & $\begin{array}{l}C_{2} \\
V_{p}\end{array}$ \\
\hline & Tilisolol & $O$-Palmitoyl tilisolol \\
\hline$K_{12}\left(\min ^{-1}\right)$ & $0.243 \pm 0.034$ & $0.351 \pm 0.250$ \\
\hline$K_{21}\left(\min ^{-1}\right)$ & $0.050 \pm 0.005$ & $0.113 \pm 0.055$ \\
\hline$K_{\mathrm{el}}\left(\min ^{-1}\right)$ & $0.083 \pm 0.015$ & $0.086 \pm 0.014$ \\
\hline$V_{\mathrm{c}}(1)$ & $0.483 \pm 0.098$ & $0.035 \pm 0.004$ \\
\hline$V_{\mathrm{p}}(1)$ & $2.27 \pm 0.35$ & $0.103 \pm 0.011$ \\
\hline$A U C(\mu \mathrm{M} \cdot \min )$ & $49.5 \pm 11.2$ & $613 \pm 93$ \\
\hline$M R T$ (min) & $83.6 \pm 11.6$ & $65.5 \pm 14.4$ \\
\hline
\end{tabular}

Each value represents the mean \pm S.E. of at least three experiments.

venous administration of the drugs presented a two-phase elimination. Therefore, the blood concentration profiles of drugs were pharmacokinetically analyzed using a two-compartment model. The calculated pharmacokinetic parameters are listed in Table 1. Distribution volumes $\left(V_{\mathrm{c}}\right.$ and $\left.V_{\mathrm{p}}\right)$ of $O-$ palmitoyl tilisolol after intravenous administration of $O$ palmitoyl tilisolol were reduced to approximately $5 \%$ compared with the distribution volume of tilisolol after the intravenous administration of tilisolol. Similarly, Tokui et al. reported that the distribution volume of the palmitoyl prodrug of rhizoxin was approximately $0.1 \%$ compared with the distribution volume of rhizoxin after the intravenous administration in mice. ${ }^{3)}$ It was previously reported that macromolecular prodrugs suppress both the distribution volume and clearance of parent compounds. ${ }^{13)}$ Palmitic acid is a biological lipid component. Therefore, it is expected that the palmitoyl functional group may enhance the affinity of the parent drug 
Table 2. Distribution Ratio between Blood Cells and Plasma of Tilisolol and $O$-Palmitoyl Tilisolol in Rat Blood at a Drug Concentration of $5 \mu \mathrm{M}$ at $37^{\circ} \mathrm{C}$

\begin{tabular}{lccc}
\hline \hline \multirow{2}{*}{ Drugs } & Blood cells (\%) & \multicolumn{2}{c}{ Plasma (\%) } \\
\cline { 3 - 4 } & & Bound fraction & Free fraction \\
\hline Tilisolol & 55.5 & 4.0 & 40.5 \\
$O$-Palmitoyl tilisolol & 95.7 & 4.3 & 0 \\
\hline
\end{tabular}

Each value represents the mean of at least three experiments.

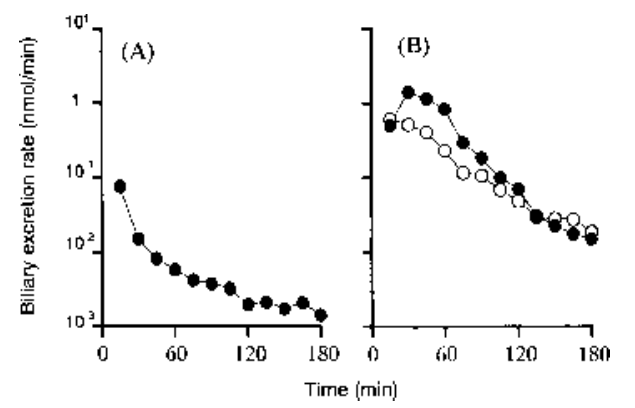

Fig. 3. Biliary Excretion Rate of Tilisolol and $O$-Palmitoyl Tilisolol after Intravenous Administration of Tilisolol (A) and $O$-Palmitoyl Tilisolol (B) at a Dose of $6 \mu \mathrm{mol} / \mathrm{kg}$ in Rats

$(\bullet)$ and $(\bigcirc)$ indicate tilisolol and $O$-palmitoyl tilisolol, respectively. Each point represents the mean \pm S.E. of at least three experiments.

on the hydrophobic components in systemic circulation, resulting in increasing their apparent molecular weight.

Table 2 lists the distribution ratios of drugs in rat blood and plasma at $37^{\circ} \mathrm{C}$ at a drug concentration of $5 \mu \mathrm{M}$. The distribution ratio between blood cells and plasma (blood/ plasma) of $O$-palmitoyl tilisolol was $95.7 \%$. However, the distribution ratio between blood cells and plasma (blood/ plasma) of tilisolol was $55.5 \%$. These results suggest that $O$ palmitoyl tilisolol exists as a form which binds with biological components, especially blood cells, in the systemic circulation. It has been reported that lipophilic drugs such as reserpine, quinidine, and cyclosporin are strongly bound to blood cells, albumin, and lipoprotein. ${ }^{14-16)}$ Suda et al. reported that lipophilic prodrugs of 5-fluorouracil were strongly bound to serum albumin in the rat blood after intravenous administration. ${ }^{17)}$ On the other hand, Tokui et al. reported that palmitoyl rhizoxin was effectively bound to LDL and was delivered to tumors via the LDL pathways. ${ }^{2,3)}$ As shown in Table 2, $O$-palmitoyl tilisolol was selectively bound to blood cells. Erythrocyte ghosts were also demonstrated to be sustained release carriers for drugs. ${ }^{18)}$ In the present study, we showed the possibility of a drug delivery system using erythrocytes by the intravenous administration of palmitoyl prodrug without preparing the drug/erythrocytes complexes before intravenous administration.

The biliary excretion rate profiles after intravenous administration of $O$-palmitoyl tilisolol and tilisolol were also examined (Fig. 3). After intravenous administration of $O$-palmitoyl tilisolol, both $O$-palmitoyl tilisolol and tilisolol were detected in bile. The biliary excretion rates of $O$-palmitoyl tilisolol and tilisolol after intravenous administration of $O$ palmitoyl tilisolol were approximately 10 - to 100 -fold higher than those of tilisolol after the intravenous administration of
Table 3. Recovery in Bile and Urine until 180 min after Intravenous Administration of Tilisolol and $O$-Palmitoyl Tilisolol in Rats

\begin{tabular}{lcc}
\hline \multicolumn{1}{c}{ Drugs } & Bile (\%) & Urine (\%) \\
\hline Tilisolol & $0.13 \pm 0.02$ & $44.6 \pm 6.8$ \\
$O$-Palmitoyl tilisolol & $2.14 \pm 0.36$ & $0.015 \pm 0.005$ \\
Tilisolol from $O$-palmitoyl tilisolol & $6.60 \pm 2.06$ & $2.20 \pm 0.67$ \\
\hline
\end{tabular}

Each value represents the mean \pm S.E. of at least three experiments.

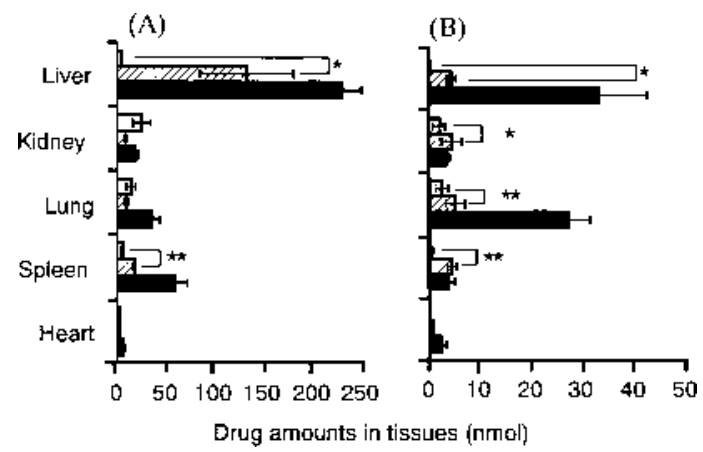

Fig. 4. Biodistribution in Tissue at $30 \mathrm{~min}$ (A) and $180 \mathrm{~min}$ (B) after Intravenous Administration of Tilisolol and $O$-Palmitoyl Tilisolol at a Dose of $6 \mu \mathrm{mol} / \mathrm{kg}$ in Rats

$(\square)$, (ם), and $(\square)$ indicate tilisolol, $O$-palmitoyl tilisolol, and tilisolol from $O$ palmitoyl tilisolol, respectively. Statistical comparisons were performed by analysis of variance $(* p<0.05, * * p<0.01)$. Each value represents the mean \pm S.E. of at least three experiments.

tilisolol. Table 3 shows the recovery of drugs in bile and urine after the intravenous administration of drugs until 180 min. Biliary recovery of $O$-palmitoyl tilisolol and tilisolol after intravenous administration of $O$-palmitoyl tilisolol was 16-fold and 51-fold higher than that of tilisolol after intravenous administration of tilisolol, respectively.

After the intravenous administration of tilisolol, most of the tilisolol was recovered in the urine (Table 3). In contrast, $O$-palmitoyl tilisolol and tilisolol after intravenous administration of $O$-palmitoyl tilisolol were scarcely recovered in the urine. The glomerular filtration of drugs was dependent on their molecular weights. ${ }^{14)}$ Therefore, the binding of $O$ palmitoyl tilisolol to biological components may suppress the glomerular filtration of $O$-palmitoyl tilisolol.

Figure 4 shows the drug amounts in various tissues after intravenous administration of tilisolol and $O$-palmitoyl tilisolol at $30 \mathrm{~min}(\mathrm{~A})$ and $180 \mathrm{~min}(\mathrm{~B})$. The amounts of $O$ palmitoyl tilisolol and tilisolol in the liver and spleen after intravenous administration of $O$-palmitoyl tilisolol were significantly higher than that of tilisolol after intravenous administration of tilisolol at 30 and $180 \mathrm{~min}$, respectively. The high amounts of $O$-palmitoyl tilisolol in these tissues after intravenous administration of $O$-palmitoyl tilisolol may be due to their high blood concentrations (Fig. 2). Therefore, the tissue uptake clearances of tilisolol and $O$-palmitoyl tilisolol after intravenous administration were calculated (Table 4). The hepatic uptake clearance of $O$-palmitoyl tilisolol was 3.6-fold higher than that of tilisolol. Thus, the tissue uptake clearance analysis also demonstrated that $O$-palmitoyl tilisolol was mainly eliminated from the liver, although large clearances of tilisolol were observed in the kidney, lung and spleen.

In summary, we examined the biodistribution and pharma- 
Table 4. $A U C$ and Tissue Uptake Clearances of Tilisolol and $O$-Palmitoyl Tilisolol after Intravenous Administration in Rats

\begin{tabular}{|c|c|c|c|c|c|c|}
\hline \multirow{2}{*}{ Drugs } & \multirow{2}{*}{$\begin{array}{c}\text { Blood } A U C \\
(\mu \mathrm{M} \cdot \min )\end{array}$} & \multicolumn{5}{|c|}{ Tissue uptake clearance $(\mu 1 / \min )$} \\
\hline & & Liver & Kidney & Lung & Spleen & Heart \\
\hline Tilisolol & 17.4 & 240 & 1500 & 840 & 338 & 131 \\
\hline$O$-Palmitoyl tilisolol & 265 & 863 & 66.9 & 139 & 223 & 24.1 \\
\hline
\end{tabular}

$A U C$ and tissue uptake clearances were calculated for the initial phase until $30 \mathrm{~min}$ after intravenous administration.

cokinetics of $O$-palmitoyl tilisolol. The blood concentrations of $O$-palmitoyl tilisolol after intravenous administration of $O$ palmitoyl tilisolol were significantly higher compared with those of tilisolol after intravenous administration of tilisolol. In addition, the hepatic uptake clearance of $O$-palmitoyl tilisolol after intravenous administration of $O$-palmitoyl tilisolol was 3.6-fold higher than that of tilisolol after the intravenous administration of tilisolol. In the in vitro experiments, it was demonstrated that the distribution ratios between blood cells and plasma (blood/plasma) of $O$-palmitoyl tilisolol and tilisolol was 95.7 and $55.5 \%$, respectively. These findings suggested that $O$-palmitoyl tilisolol exists as a binding form with biological components, especially blood cells, in systemic circulation. In conclusion, the palmitoyl prodrug approach appears to be a useful drug delivery system to deliver the parent drug to the liver.

Acknowledgments The authors thank Akemi Oshita and Chie Nakatsukasa for technical assistance. The authors also thank Nisshin Flour Milling Co., Ltd. (Tokyo, Japan) for supplying tilisolol. This work was supported in part by a Grantin-Aid for Scientific Research from the Ministry of Education, Culture, Sports, Science, and Technology, Japan.

\section{REFERENCES}

1) Bijsterbosch M. K., van de Bilt H., van Berkel T. J. C., Biochem. Pharmacol., 52, 113-121 (1996).

2) Tokui T., Takatori T., Shinozaki N., Ishigami M., Shiraishi A., Ikeda T., Tsuruo T., Cancer Chemother. Pharmacol., 36, 1-6 (1995).

3) Tokui T., Maeda N., Kuroiwa C., Sasagawa K., Inoue T., Kawai K., Ikeda T., Komai T., Pharm. Res., 12, 370-375 (1995).

4) Kawakami S., Munakata C., Fumoto S., Yamashita F., Hashida M., J. Drug Target., 8, 137-142 (2000).

5) Sasaki H., Kakutani T., Hashida M., Kimura T., Sezaki H., Chem. Pharm. Bull., 33, 2968-2973 (1985).

6) Takino T., Koreeda N., Nomura T., Sakaeda (née Kakutani) T., Yamashita F., Takakura Y., Hashida M., Biol. Pharm. Bull., 21, 492-497 (1998).

7) Tokunaga Y., Iwasa T., Fujisaki J., Sawai S., Kagayama A., Chem. Pharm. Bull., 36, 3557-3564 (1988).

8) Sqalli-Houssaini H., Pierlot C., Kusnierz J.-P., Parmentier B., MartinNizard F., Lestavel-Delattre S., Tartar A., Fruchart J.-C., Sergheraert C., Duriez P., Biotech. Ther., 5, 69-85 (1994).

9) Kawakami S., Yamamura K., Mukai T., Nishida K., Nakamura J., Sakaeda T., Nakashima M., Sasaki H., J. Pharm. Pharmacol., 53, 1157-1161 (2001)

10) Kawakami S., Nishida K., Mukai T., Yamamura K., Kobayashi K., Sakaeda T., Nakamura J., Nakashima M., Sasaki H., J. Pharm. Sci., 90, 2113-2120 (2001)

11) Nakagawa Y., Sugai T., Chin W. P., Shibuya T., Hashimoto K., Imai S., Arzneimittelforschung, 34, 194-199 (1984).

12) Yamaoka K., Tanigawara Y., Nakagawa T., Uno T., J. PharmacobioDyn., 4, 879-885 (1981).

13) Takakura Y., Takagi A., Hashida M., Sezaki H., Pharm. Res., 4, 293 300 (1987).

14) Lemaire M., Tillement J. P., J. Pharm. Pharmacol., 34, 715-718 (1982).

15) Nilsen O. G., Biochem. Pharmacol., 25, 1007-1012 (1976).

16) Chen Z., Danon A., Biochem. Pharmacol., 28, 267-271 (1979).

17) Suda Y., Shimidzu K., Sumi M., Oku N., Kusumoto S., Nadai T., Yamashita S., Biol. Pharm. Bull., 16, 876-878 (1993).

18) Ohsako M., Oka Y., Tsuzuki O., Matsumoto Y., Biol. Pharm. Bull., 18, 310-314 (1995). 